\title{
Neural patterns during anticipation predict emotion regulation success for reappraisal
}

\author{
Elektra Schubert ${ }^{1} \cdot$ James A. Agathos ${ }^{1,2} \cdot$ Maja Brydevall $^{1} \cdot$ Daniel Feuerriegel ${ }^{1} \cdot$ Peter Koval $^{1,3} \cdot$ Carmen Morawetz $^{4}$. \\ Stefan Bode ${ }^{1}$
}

Published online: 6 July 2020

(C) The Psychonomic Society, Inc. 2020

\begin{abstract}
The ability to exert control over emotions, termed emotion regulation (ER), is vital for everyday functioning. ER success may be influenced by processes relating to the anticipation (prior to active regulation) and implementation (during active regulation) of ER strategy use. We investigated whether brain activity patterns recorded using electroencephalography (EEG) during the first second of anticipation and implementation of two ER strategies - distraction and reappraisal - were related to regulation success. Participants viewed negative images that evoked disgust and sadness. Before each image was presented, participants were cued to either passively view the image or decrease their emotional responses. ER success scores were calculated from subsequent selfreported disgust and sadness ratings. Using multivariate support vector regression, ER success scores were predicted from spatiotemporal patterns of event-related potentials during the first second of anticipation and implementation phases of each ER strategy. For both sadness and disgust, reappraisal success could be predicted during anticipation, while distraction success could be predicted during implementation. These findings suggest that early anticipatory cognitive processes are a key determinant of reappraisal success, but may not be similarly important for distraction. This may be because reappraisal is more cognitively demanding than distraction, requiring enhanced preparation of mental resources.
\end{abstract}

Keywords Emotion regulation $\cdot$ Regulation success $\cdot$ Anticipation $\cdot$ Electroencephalography $(E E G) \cdot$ Multivariate pattern analysis (MVPA)

Human emotions serve important functions, such as helping orient attention toward salient changes in the environment and preparing the body to respond (Thompson, 1994). However, to be useful for adaptive behaviour, emotions sometimes must be regulated (Gross, 1998). For example, we can calm ourselves down when feeling sad and prevent ourselves from

Electronic supplementary material The online version of this article (https://doi.org/10.3758/s13415-020-00808-2) contains supplementary material, which is available to authorized users.

Stefan Bode

sbode@unimelb.edu.au

1 Melbourne School of Psychological Sciences, The University of Melbourne, Parkville, Victoria 3010, Australia

2 Department of Psychiatry, The University of Melbourne, Parkville, Australia

3 KU Leuven-University of Leuven, Leuven, Belgium

4 Center for Medical Physics and Biomedical Engineering, Medical University Vienna, Wien, Austria lashing out when angered or disgusted. Impaired ability to control one's emotions is linked to poor well-being and social functioning, lower resilience to stressful life events, and psychological disorders (e.g., Beauregard, Paquette, \& Levesque, 2006; Kanske, Heissler, Schönfelder, \& Wessa, 2012; Koole, 2009; Troy, Wilhelm, Shallcross, \& Mauss, 2010). Greater emotion regulation (ER) ability, on the other hand, has been linked with higher life satisfaction and mental well-being (Côté, Gyurak, \& Levenson, 2010). Hence, it is important to understand the behavioural and neural mechanisms through which emotions are controlled. An important aspect that is still poorly understood is whether the success of ER depends on one's ability to mentally prepare for using a specific strategy - in particular, the role of fast processes immediately after learning that a negative emotional event will subsequently occur (Shafir, Schwartz, Blechert, \& Sheppes, 2015).

Emotions involve changes in subjective experience, behaviour, and physiology (Gross \& Levenson, 1997). Although both positive and negative emotions can be maladaptive if dysregulated, much of the ER literature focuses on regulation of negative emotions. This focus may be warranted given a 
recent meta-analysis, which found that the dynamics of negative emotions are more strongly associated with clinical disorders and well-being than positive emotion dynamics (Houben, Van Den Noortgate, \& Kuppens, 2015). Therefore, in the current study, we aimed to explore the neural underpinnings of the successful regulation of negative emotions - specifically, sadness and disgust. Experiencing sadness is a normal part of life; however, excessive sadness may be linked to poorer social relations and well-being, as well as depression (Holtzheimer \& Mayberg, 2011; Rivers, Brackett, Katulak, \& Salovey, 2007). Disgust is important for avoiding pathogens and contaminants (Oaten, Stevenson, \& Case, 2009) and plays an important role in complex social processes, such as moral decision-making (Ivan, 2015). However, the excessively frequent or intense experience of disgust can become maladaptive to overall psychological health, even pathological as in obsessive-compulsive disorder (Fink, Pflugradt, Stierle, \& Exner, 2018; Olatunji, Berg, \& Zhao, 2017), and can lead to social biases and prejudice towards outgroups (Liu, Lin, Xu, Zhang, \& Luo, 2015). The ability to regulate sadness and disgust is therefore important for maintaining healthy functioning (e.g., Davis, QuiñonesCamacho, \& Buss, 2016; Szasz, Hofmann, Heilman, \& Curtiss, 2016). As sadness and disgust are distinct from one another, with little overlap (Cowen \& Keltner, 2017), it is beneficial to study them separately. We reasoned that since disgust is typically considered a high-arousal emotion and sadness a low-arousal emotion (Posner, Russell, \& Peterson, 2005; Russell, 1980), and disgust is typically recognized more easily and unfolds more rapidly than sadness (Costa et al., 2014; Keltner, Sauter, Tracy, \& Cowen, 2019; Verduyn \& Lavrijsen, 2015), studying these two emotions might also allow for testing whether the importance of preparing for emotion regulation may depend on the specific emotion.

ER is a set of extrinsic and intrinsic goal-directed processes used for monitoring, evaluating, and modifying how emotions unfold over time (Gross, 2002; Gross, 2015; Gyurak, Gross, \& Etkin, 2011; Koole, 2009; McRae et al., 2010; Thompson, 1994). Two well-studied ER strategies are distraction and reappraisal (e.g., McRae, Ciesielski, \& Gross, 2012; Shafir et al., 2015; Thiruchselvam, Blechert, Sheppes, Rydstrom, \& Gross, 2011; Webb, Miles, \& Sheeran, 2012). Distraction involves diverting attention away from the emotional stimulus, before the stimulus is appraised, often by explicitly generating neutral thoughts (e.g., thinking about daily activities) unrelated to the emotional stimulus (Gross, 2001; McRae et al., 2010; Webb et al., 2012). Reappraisal involves reinterpreting the meaning of an emotional situation or stimulus to decrease its emotional impact (e.g., imagining that a bad situation will improve over time; e.g., Thiruchselvam et al., 2011; Webb et al., 2012). Distraction and reappraisal differ in many ways, including their effectiveness for emotional situations with different intensity (Shafir et al., 2015; Sheppes, Brady, \&
Samson, 2014), timing and required cognitive effort (Sheppes \& Gross, 2011; Sheppes \& Meiran, 2007; Strauss, Ossenfort, \& Whearty, 2016; Thiruchselvam et al., 2011), and physiological and neural mechanisms (Dörfel et al., 2014; Kanske, Heissler, Schönfelder, Bongers, \& Wessa, 2011; Ochsner et al., 2004; Sheppes, Catran, \& Meiran, 2009).

Another important difference between these strategies may be whether they benefit from being anticipated. Recent studies investigating anticipation have mostly focused on preparing for ER depending on the expected emotional intensity of an upcoming stimulus. Shafir et al. (2015) showed that anticipating high or low intensity stimuli resulted in differential preferences for ER strategies, with distraction being the preferred strategy for high intensity stimuli and reappraisal for low intensity stimuli (also see Sheppes \& Levin, 2013; Sheppes, Scheibe, Suri, \& Gross, 2011). Interestingly, participants still preferred receiving anticipatory information about the stimuli even when explicitly instructed to use a strategy for which this information was not helpful (Shafir \& Sheppes, 2018). This suggests that people often perceive anticipatory information about an emotion regulation scenario as desirable. Denny, Ochsner, Weber, and Wager (2014) reanalyzed data from a functional magnetic resonance imaging (fMRI) study (Wager et al., 2008) in which participants were instructed to anticipate using reappraisal versus not regulating. Activation in prefrontal and subcortical brain regions during reappraisal anticipation was selectively associated with changes in amygdala activity, which, in turn, was associated with ER success. Another study measured the stimulus-preceding negativity (SPN), an event-related potential (ERP) component preceding the appearance of a stimulus, and found that SPN amplitudes were higher during reappraisal anticipation relative to a no regulation condition (Moser, Krompinger, Dietz, \& Simons, 2009; for similar findings, see also Shafir et al., 2015; Thiruchselvam et al., 2011). Taken together, these studies suggest a tight link between recruiting cognitive resources during anticipation and ER success.

However, it remains unclear whether ER anticipation is equally important for strategies other than reappraisal that are less complex and might be less dependent on the early recruitment of cognitive resources. In addition, in previous studies, anticipation usually comprised a prolonged time period in which a mixture of fast, potentially automatic and deliberate, controlled cognitive processes could have taken place. Our aim for this study was to isolate the neural mechanisms underlying cognitive processes during the first second of ER anticipation (which contained the typical time window of several ERP components, such as the N1, P1, P3, SPN, and LPP), and compare them to those observable during the first second of ER implementation. Using EEG, we investigated whether trial-by-trial differences in ER success for emotionally evocative stimuli could be predicted from neural activity patterns emerging during the first second of the anticipation and 
implementation of both distraction and reappraisal. We used multivariate pattern analysis (MVPA; Bode, Feuerriegel, Bennett, \& Alday, 2019), which offers a novel way to study fast neural processes immediately following a stimulus (e.g., Bode, Bennett, Stahl, \& Murawski, 2014). MVPA uses data from all electrodes, rather than a single electrode or region of interest, and it is not dependent on the presence of specific patterns of event-related potentials at preselected electrodes. The unique sensitivity of MVPA may therefore allow for the detection of subtle differences between experimental conditions that may be overlooked by conventional, univariate methods (Bode et al., 2019).

\section{Methods}

\section{Participants}

Thirty-nine participants were recruited. Participants were right-handed, fluent in English, had normal or corrected-tonormal vision, and no history of mental illness. Two participants were excluded for failing to correctly remember the symbols used to index the ER strategies (see below). In order for the cross-validation procedure to run properly (see below), participants were required to have at least 20 artefact-free trials remaining in each analysis condition (distraction anticipation, distraction implementation, reappraisal anticipation, reappraisal implementation) after EEG preprocessing. Ten participants were excluded for failing to meet this criterion. No significant differences in any variables or questionnaire items were found between excluded participants versus those included in the final analyses (see Supplementary Table S1). The final sample comprised 27 participants, ages 18 to 37 years ( $M=22.48$ years, $S D=3.95 ; 20$ females, seven males). This is slightly larger than the sample size typically used in similar EEG studies (e.g., 15-20 participants; Uusberg, Thiruchselvam, \& Gross, 2014; Moser, Hajcak, Bukay, \& Simons, 2006; Thiruchselvam et al., 2011). Participants gave written informed consent, were debriefed after completing the task, and compensated AUD\$25 for their time. The experiment was conducted in accord with the Declaration of Helsinki and approved by the Human Research Ethics Committee of the University of Melbourne (ID 1851914).

\section{Questionnaires}

Several questionnaires were used to determine whether, on average, the sample differed from population averages on alexithymia or particular ER tendencies. Participants completed the 20-item Toronto Alexithymia Scale (TAS-20; Bagby, Parker, \& Taylor, 1994) testing for alexithymia, an inability to identify and describe one's emotions. Six items from the Emotion Regulation Questionnaire (ERQ; Gross \& John,
2003) assessed the tendency to habitually use cognitive reappraisal. Twelve items from the Regulation of Emotion Systems Survey (RESS; De France \& Hollenstein, 2017) examined tendencies to habitually use distraction and reappraisal in response to negative emotions.

\section{Stimuli}

Forty negative images (mean valence rating $<4$ out of 7 , according to norms; Riegel et al., 2016) related to sadness and disgust were obtained from the Nencki Affective Picture System (NAPS; Marchewka, Żurawski, Jednoróg, \& Grabowska, 2014) and resized to $400 \times 300$ pixels. In order to obtain these images, "sadness specificity" and "disgust specificity" scores were computed for every image in the database by calculating the sum of differences between its ratings (Riegel et al., 2016) for (a) sadness or (b) disgust, and for other negative emotions (fear, surprise, anger, and either disgust or sadness, respectively). The 20 images with the highest sadness and disgust specificity scores were then used in the experiment. Note that stimuli nevertheless did not trigger single emotions, but most likely always a mixture of emotions.

\section{Emotion regulation cues}

In each trial, participants were cued with an abstract symbol indicating whether to employ one of the two ER strategy or passive viewing in that trial (see Fig. 1a). The three conditions were "look" (no regulation; allowing emotions to naturally unfold in response to the image), "distract" (thinking about something unrelated to the image), and "reappraise" (imagining that the depicted situation would improve, or focusing on its positive aspects). Symbols were neutral, unrelated to the specific upcoming emotional stimuli, and free from religious or cultural connotations. Six symbols were used for each participant; two symbols represented the look instruction, two symbols represented the distract instruction, and two symbols represented the reappraisal instruction. Allocation of symbols to instructions was randomly counterbalanced across participants. Two randomly allocated symbols for each instruction were used to ensure that the neural representations were truly associated with the preparation for each strategy, rather than visual or semantic processing of a word (e.g., "distract") or one specific symbol.

\section{Procedure}

Participants first completed the questionnaires before the task was conducted on a standard Dell PC interfacing a Dell P2210 monitor (screen resolution $1,680 \times 1,050$ pixels, frame rate 60 $\mathrm{Hz}$ ). Stimuli were presented using Psychtoolbox (Version 3; Kleiner et al., 2007) and MATLAB (R2017a). 


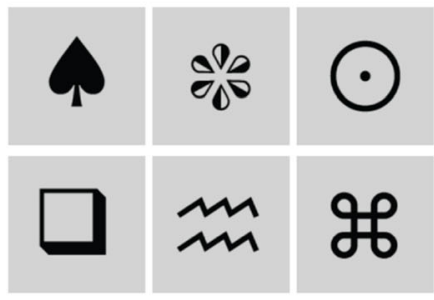

b

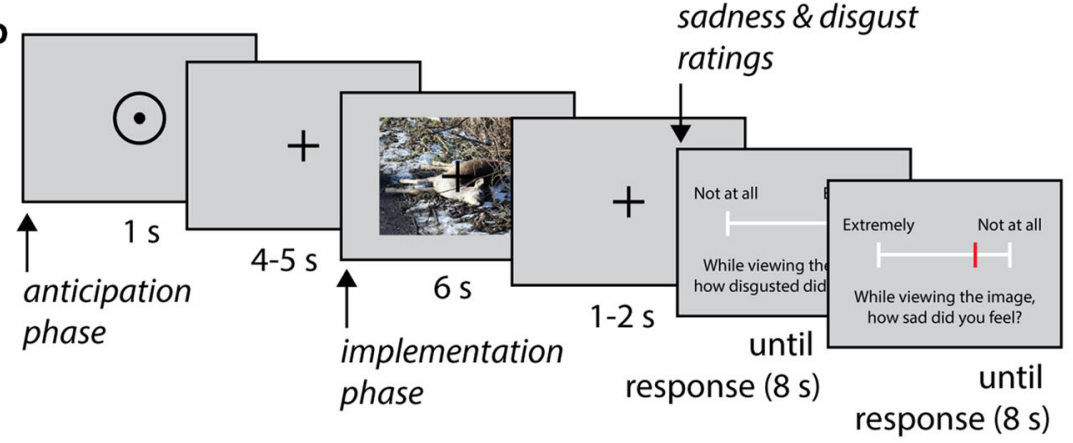

C

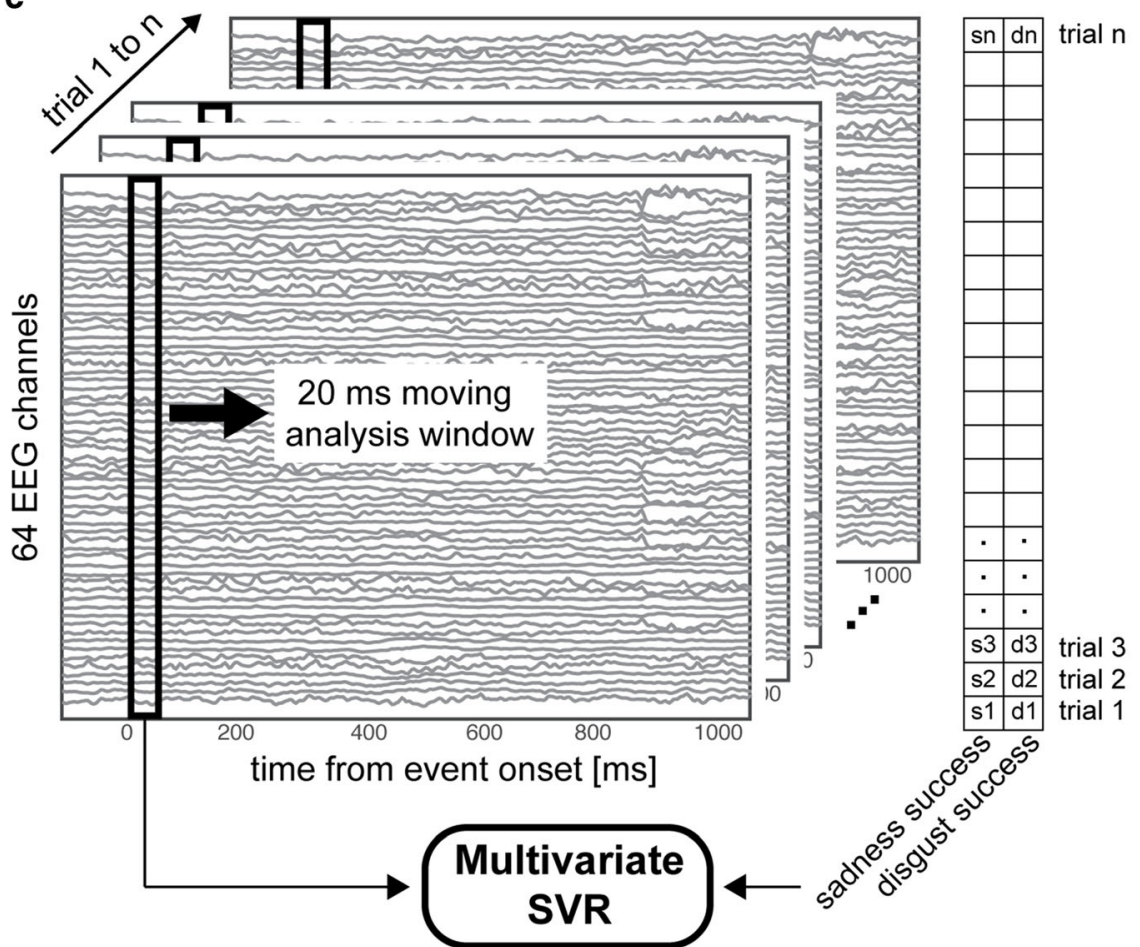

d

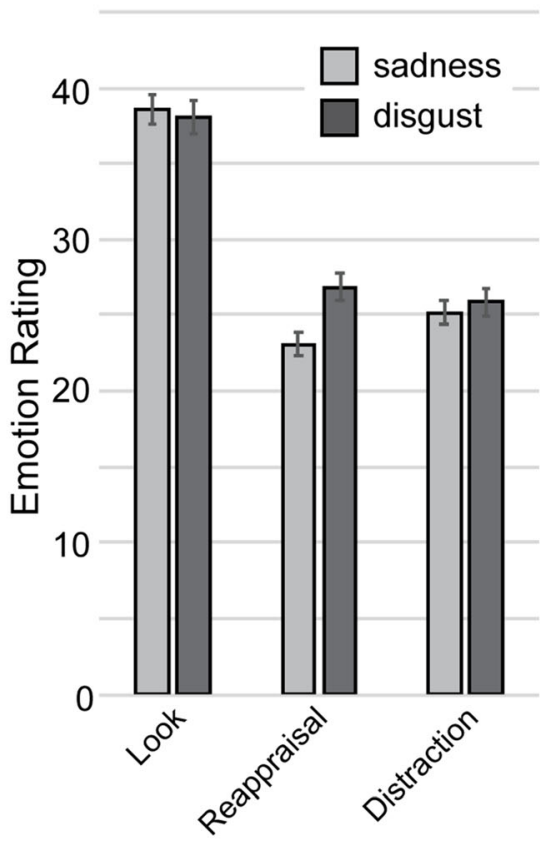

Fig. 1 Experimental paradigm, pattern classification approach, and emotion scores. a Six distinct abstract symbols were used, two of which were cues for the same instruction. The assignment of cues to instructions (distraction, reappraisal, look) was pseudorandomized across participants. b In each trial, participants were first shown a strategy cue (anticipation phase). After a delay, they were then presented with a negative image and applied the cued strategy (implementation phase). This was followed by two emotion rating screens, asking participants to state how sad and disgusted they felt at the end of the trial. The order of rating questions and scale end points was randomized across trials. c
Multivariate support vector regression (SVR) procedure. Spatiotemporal data $(20 \mathrm{~ms})$ were extracted using a moving analysis time window for each trial and transformed into pattern vectors. A linear SVR classifier was trained on $80 \%$ of the data to estimate a regression model predicting each trial's success scores for regulating sadness and disgust. Unseen data (20\%) were used for testing, and the correlation between predicted and actual success scores after 10 iterations of the five-fold cross-validation procedure (50 tests) was calculated for each time window. Results were statistically tested against an empirical chance distribution. d Emotion rating scores for sadness and disgust by emotion regulation strategy
In a practice session, participants first learned to employ each strategy. They completed three trials per strategy in which a cue word (e.g., "distract") appeared for $1 \mathrm{~s}$, followed by a negative image (which did not feature in the main study), which remained until the participant pressed the space bar. While viewing the image, the participant was asked to describe their ER strategy aloud. Next, participants were trained to associate symbols with specific ER strategies and were subsequently tested on their knowledge of the symbolstrategy associations. The training concluded when participants gave no incorrect responses in two consecutive iterations of the test, which included all symbols twice. Finally, participants performed six practice trials, identical to the main experiment, but viewing stimuli that did not feature in the main experiment.

In the experiment, each trial began with an ER anticipation phase: First, a strategy symbol was presented for $1 \mathrm{~s}$, followed by a fixation cross between $4 \mathrm{~s}$ to $5 \mathrm{~s}$, allowing for mental preparation of the ER strategy, but participants were explicitly instructed not to start employing it (e.g., starting to distract 
themselves). This was followed by the ER implementation phase, during which one of the negative images was presented for $6 \mathrm{~s}$, again followed by a fixation cross for (1-2 s). Participants were instructed to experience (look condition) or regulate their emotions (distraction and reappraisal conditions). Participants then answered two questions: "How sad do you feel?" and "How disgusted do you feel?" The order of these questions was randomized across trials. Participants used a computer mouse to move a bar on a continuous rating scale that appeared under the text (ranging from "not at all" on one end to "extremely" at the other) and clicked the mouse to make their response. If no click was made after $8 \mathrm{~s}$, the bar's final position was recorded as the response. This occurred on 61 out of 3,241 trials for the disgust scale, and 76 out of 3,241 trials for the sadness scale. Thus, missing responses were replaced on approximately $2 \%$ of all trials. To minimize order effects and ensure participants were paying attention, the end points of the scale in both the practice trials and main task were randomized across trials (see Fig. 1b). Participants were informed of this at the beginning of the experiment. Participants then saw a fixation cross for $1-2 \mathrm{~s}$ before the next trial started. All fixation durations were randomly drawn from uniform distributions (250 ms steps) to prevent temporal expectation effects.

The experiment comprised 120 trials. Each image was presented three times (each time paired with a different instruction) in a randomized order. Multiple image presentation ensured that results from the three conditions were comparable. Breaks occurred every 30 trials, during which participants could relax until ready to continue. Following the experiment, participants were tested again on each symbol cue, to ensure they had been using them correctly. Participants who failed this test were excluded from analyses $(n=2)$. On average, the experiment took approximately 1.5 hours, including EEG setup and pack-down.

\section{EEG recording and data preprocessing}

Electrophysiological activity was recorded during the ER task using a 64-channel BioSemi Active II system (sample rate 512 $\mathrm{Hz}$, recording bandwidth DC-102 Hz). The system comprised $64 \mathrm{Ag} / \mathrm{AgC} 1$ electrodes, attached to a fabric cap according to the International 10-20 System. Four additional electrodes were placed next to and below the left eye, recording the horizontal and vertical electrooculogram, as well as above the left and right mastoids. Electrode offsets were kept within $\pm 50 \mu \mathrm{V}$.

EEG data were processed using EEGLab (Version 14.1.2; Delorme \& Makeig, 2004). Data were rereferenced to the average of left and right mastoids. Data were high-pass and low-pass filtered at $0.1 \mathrm{~Hz}$ and $30 \mathrm{~Hz}$, respectively (EEGLab FIR Filter New, default settings). Data were segmented into epochs, beginning $100 \mathrm{~ms}$ prior to either appearance of an ER strategy cue (anticipation phase) or image presentation (implementation phase), and ending 1,000 $\mathrm{ms}$ after each event to capture initial periods of cognitive activity related to each phase. Epochs were baseline-corrected using the preevent period. Data were then visually inspected for muscle and skin potential artefacts and removed if contaminated. Channels with excessively noisy signals were removed and interpolated using spherical spline interpolation (average number of channels interpolated $=0.93$, range: $0-7$ ). An independent component analysis (ICA), as implemented in EEGLab, was performed to identify and remove eye movements, saccades, and blinks. Epochs were excluded from analyses if the amplitudes at any channel exceeded $\pm 150 \mu \mathrm{V}$.

\section{Data analysis}

\section{Success scores}

For each distraction and reappraisal trial, a measure of ER success was calculated (henceforth referred to as "success score"; Morawetz, Alexandrowicz, \& Heekeren, 2017). First, the mean sadness and disgust ratings from all Look trials were calculated for each participant (Sadness $_{\text {Look_Mean }}$, Disgust $_{\text {Look_Mean }}$ ) to obtain baseline levels of sadness and disgust evoked when no ER strategy was applied. This approach, similar to that taken in previous studies (Morawetz et al., 2017; Morawetz, Bode, Baudewig, Jacobs, \& Heekeren, 2016; Morawetz, Mohr, Heekeren, \& Bode, 2019; Wager et al., 2008), was used to average out situational variations between individual Look trials (which were expected, given the event-related nature of the task) and to minimize order effects. The success scores for sadness and disgust for a particular trial were then calculated separately by using the sadness and disgust ratings $\left(\right.$ Sadness $_{\text {Trial_X }}$, Disgust ${ }_{\text {Trial }} \mathrm{X}$ ) for the given trial, as,

$$
\begin{aligned}
& \text { Sadness_Success } \text { trial_x }_{-x}=\frac{\text { Sadness }_{\text {Look }_{\text {mean }}}-\text { Sadness }_{\text {trial_x }}}{\text { Sadness }_{\text {Look }_{\text {mean }}}} ; \\
& \text { Disgust_Success }_{\text {trial }_{x}}=\frac{\text { Disgust }_{\text {Look }_{\text {mean }}}-\text { Disgust }_{\text {trial }}}{\text { Disgust }_{\text {Look }_{\text {mean }}}}
\end{aligned}
$$

These success scores reflected the percentage reduction in sadness/disgust ratings after distraction or reappraisal relative to a participant's average unregulated sadness/disgust. For example, a sadness success score of 1 would indicate that on this particular trial, using the cued ER strategy reduced $100 \%$ of the sadness usually experienced on look trials, whereas 0.1 would indicate a $10 \%$ reduction.

\section{Multivariate pattern analyses of EEG data}

The association between brain activity and ER success scores on each distraction and reappraisal trial was investigated using 
linear support vector regression (SVR), conducted using the Decision Decoding Toolbox (Version1.0.3; DDTBOX; Bode et al., 2019). For each participant, the analysis was conducted for the anticipation and implementation phases separately. Using a "sliding window" approach, an analysis time window of nonoverlapping 20-ms steps was moved through the data, covering the entire epoch. Within each analysis window, all ten data points from all 64 EEG channels were transformed into vectors, representing the spatiotemporal brain activity patterns $(10 \times 64=640$ features $)$ associated with each trial. Because only trials in which distraction or reappraisal were employed were of interest, trials from the look condition were discarded. This means that each trial's activity pattern was associated with one sadness success score and one disgust success score. Each time window's multivariate activity pattern was then used independently to predict sadness and disgust success scores, separately for distraction and reappraisal conditions. For this, a linear SVR model (using a standard cost parameter $\mathrm{C}=0.1$, interfacing LIBSVM; Chang \& Lin, 2011) was trained on $80 \%$ of the data, which was randomly selected to estimate the model, and then tested on the remaining $20 \%$ (see Fig. 1c). This process was repeated independently using a five-fold cross-validation procedure until all data sets had been used as test data once. This cross-valiadition analysis was itself repeated 10 times, using newly drawn random data, to obtain a conservative estimate of decoding performance based on the average performance of the classifier from all cross-validation steps and analysis repetitions, circumventing drawing biases.

The outcome for each analysis time window was an average Fisher Z-transformed correlation coefficient between the predicted and the true success scores, separately for both ER strategies (distraction and reappraisal) and both emotions (sadness and disgust). To estimate an empirical chance distribution of results, the procedure was repeated using the same data in an identical fashion, but with success scores (labels) randomly shuffled across trials (cf. Bode et al., 2014; Bode \& Stahl, 2014; Chan, Pianta, Bode, \& McKendrick, 2017; Siswandari, Bode, \& Stahl, 2019; Turner, Johnston, de Boer, Morawetz, \& Bode, 2017). For group-level statistical analyses, the empirical results were tested against the shuffled-label/chance results for each analysis time window. Corrections for multiple comparisons were performed using cluster-based permutation tests based on the cluster mass statistic (cluster inclusion alpha $=0.05$, permutation samples $=5,000)$. This approach takes advantage of the statistical nonindependence of adjacent analysis time windows and is not as overly conservative as the Bonferroni correction.

Finally, as a control analysis, DDTBOX was also used to perform linear support vector classification analyses to predict the ER strategies from activity patterns. For this, each trial was sorted into one of three ER conditions (distraction, reappraisal, look). Using the same cross-validation procedure described above, each analysis time window (both during anticipation and implementation) was used to predict which strategy participants were employing on that trial. We expected that the strategy which allowed for prediction of ER success in the respective task phase would also be decodable from the look condition during the same phase.

\section{Results}

\section{Sample characteristics}

First, we tested whether our sample significantly deviated from established norms with respect to alexithymia and habitual use of ER strategies, using the TAS-20, ERQ and RESS. Female participants were on average above the norms reported by De France and Hollenstein (2017) for the TAS$20(t=3.23, p=.004)$ as well as the RESS-Distraction scale $(t$ $=2.53, p=.021)$. However, for all scales, including the TAS20 and the RESS, on average our sample did not differ more than one standard deviation from the population averages, suggesting that our participants were not atypical or unable to perform ER (all test statistics are reported in the Supplementary Table S2). Due to the relatively small sample size, questionnaire data were not used for further analyses.

\section{Behavioural results}

\section{Emotion ratings}

First, we confirmed that the NAPS images selected to elicit sadness and disgust successfully elicited these specific emotions by examining emotion ratings in the look condition. Participants' continuous emotion ratings were converted to emotion scores from 0 (not at all) to 100 (extremely). As expected, sadness images were rated higher on sadness than disgust images, $t(38)=9.10, p<.001$, mean difference $=$ $17.95,95 \% \mathrm{CI}[13.95,21.94]$, while disgust images were rated higher on disgust than sadness images, $t(38)=10.58, p<.001$, mean difference $=26.80,95 \%$ CI $[21.67,31.93]$. Importantly, participants reported experiencing some degree of sadness and disgust for all images. A summary of sadness and disgust ratings by trial type can be found in Supplementary Table S3.

We then tested whether the use of each ER strategy successfully reduced sadness and disgust experience (see Fig. 1d). Given that all images elicited some sadness and disgust experience, these analyses were conducted for the full image set. We tested for this using one-way repeated-measures analyses of variance (ANOVAs) with Greenhouse-Geisser epsilon corrections for sphericity violations. Sadness ratings were significantly different across the three conditions, $F(1.29$, $33.50)=28.54, p<.001, \eta_{\mathrm{p}}{ }^{2}=.523$. Follow-up comparisons with Bonferroni corrections revealed that, compared with the 
look condition, sadness ratings were lower for both distraction, $t(26)=5.74, p<.001$, mean difference $=15.50,95 \% \mathrm{CI}$ $[8.59,22.407]$, and reappraisal, $t(26)=5.36, p<.001$, mean difference $=13.41,95 \%$ CI $[7.01,19.81]$. No significant difference was found between distraction and reappraisal, $t(26)=$ $1.81, p=.244$. Similarly, disgust ratings also varied across ER strategies, $F(1.21,31.44)=30.71, p<.001$. Disgust ratings were lower compared to the look condition for distraction, $t(26)=5.52, p<.001$, mean difference $=12.24,95 \% \mathrm{CI}$ $[7.68,16.79]$, and reappraisal, $t(26)=6.14, p<.001$, mean difference $=11.25,95 \%$ CI $[7.48,15.02]$. No significant difference was found between distraction and reappraisal, $t(26)=$ $1.14, p=.792$.

We also converted ratings into success scores (see Table 1). Across the sample sadness success scores were significantly different from zero, for both distraction, $t(26)=8.69, p<.001$, and reappraisal, $t(26)=8.90, p<.001$. Disgust success scores were also significantly different from zero, for both distraction, $t(26)=4.53, p<.001$, and reappraisal, $t(26)=2.86, p=$ .008 . Additionally, compared with disgust, the success scores for sadness had a higher mean, $t(26)=2.76, p=.010$, and lower average standard deviation, $t(26)=-2.85, p=.009$.

\section{MVPA results for EEG data}

\section{Regression of success scores}

Anticipation phase Linear SVR analyses revealed that sadness success scores could be predicted for reappraisal during multiple time windows following cue onset (200-220, 280-300, 720-740, 820-900, and 940-980 ms; see Fig. 2). No time windows showed above-chance prediction of sadness success scores for distraction. For disgust, a similar pattern emerged with marginally significant time windows for reappraisal (880-900 and 920-940 ms after cue onset; none of these survived correction for multiple comparisons) and no significant above-chance prediction for distraction (see Fig. 2; see Supplementary Tables S4 and S5 for detailed results).

Implementation phase In contrast to results from the anticipation phase, sadness success scores could be predicted for distraction during multiple time windows following stimulus onset $(280-300,360-560,580-600,640-700,740-820,840$ 900, and 920-960 ms; see Fig. 3). For disgust success scores, there were several significant time windows for distraction following stimulus onset (180-220, 360-380, 440-460; 560-620, 640-680, 740-760, and 860-880 ms), but these did not survive correction for multiple comparisons. No time windows showed significant above-chance prediction for reappraisal, for both the sadness and disgust success scores (see Fig. 3; see Supplementary Tables S4 and S5 for detailed results).

\section{Control analyses: ER strategy decoding}

We then used pair-wise multivariate classification to decode the ER strategies from the look condition based on EEG data from the same time windows. During the anticipation phase, it was possible to decode whether participants were preparing for reappraisal versus look strategies (see Supplementary Fig. S1). For distraction versus look, only a few time windows were marginally significant. During the implementation phase, it was possible to decode whether participants were preparing for distraction versus look; however, no time windows were significant for reappraisal versus look. In sum, only the ER strategy that allowed for the prediction of ER success during each specific phase was also clearly decodable from brain activity during the same task period.

\section{Discussion}

We investigated whether neural activity patterns, emerging within the first second following the presentation of an ER strategy cue, enabled prediction of trial-wise ER success. We compared two ER strategies, distraction and reappraisal, and validated the results using two distinct emotions, sadness and disgust. Note that since both emotions showed similar patterns, these will be discussed together here, although the results for disgust were only marginally significant (potentially due to the differences in mean and standard deviation between both emotions). We found that neural patterns immediately after ER strategy cueing, long before the emotioninducing stimulus was presented, predicted the success of reappraisal but not of distraction. Conversely, the success of distraction but not reappraisal could be predicted by neural patterns occurring directly after presentation of negative emotional stimuli. This pattern of results was mirrored in

Table 1 Emotion rating success scores for sadness and disgust by regulation strategy

\begin{tabular}{llll}
\hline & Mean sadness success score & $S D$ sadness success score & Mean disgust success score \\
\hline Reappraisal & 0.41 & 0.24 & 0.22 \\
Distraction & 0.47 & 0.28 & 0.34 \\
\hline
\end{tabular}

Note. $S D=$ standard deviation 


\section{Anticipation}

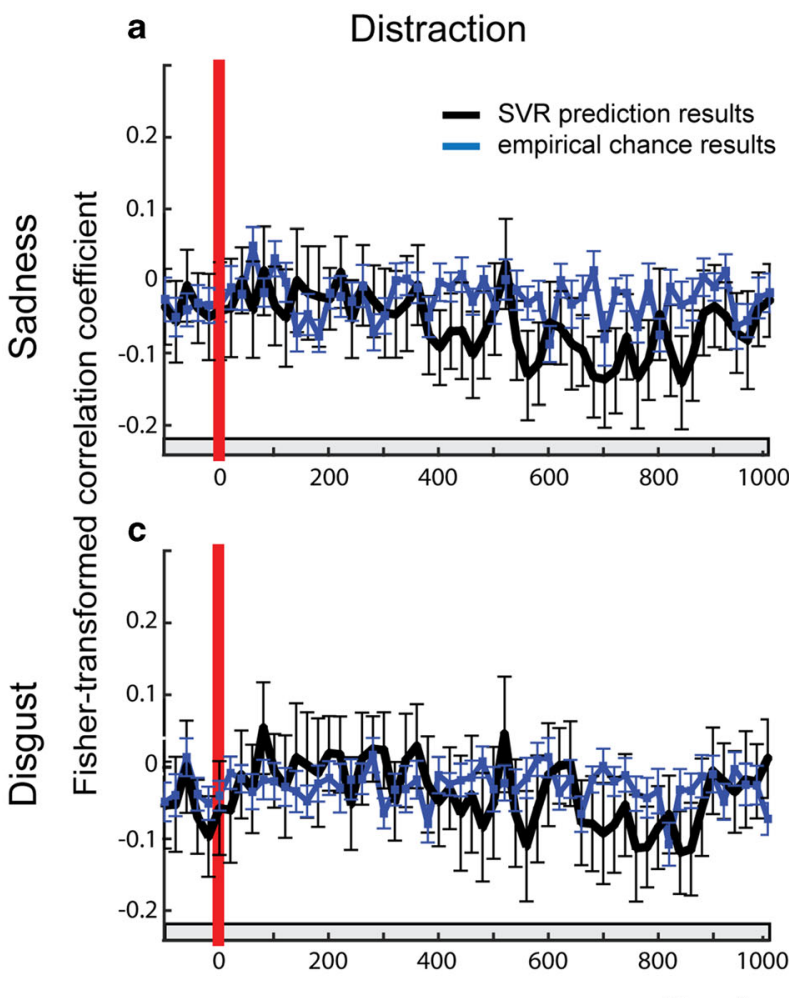

b Reappraisal
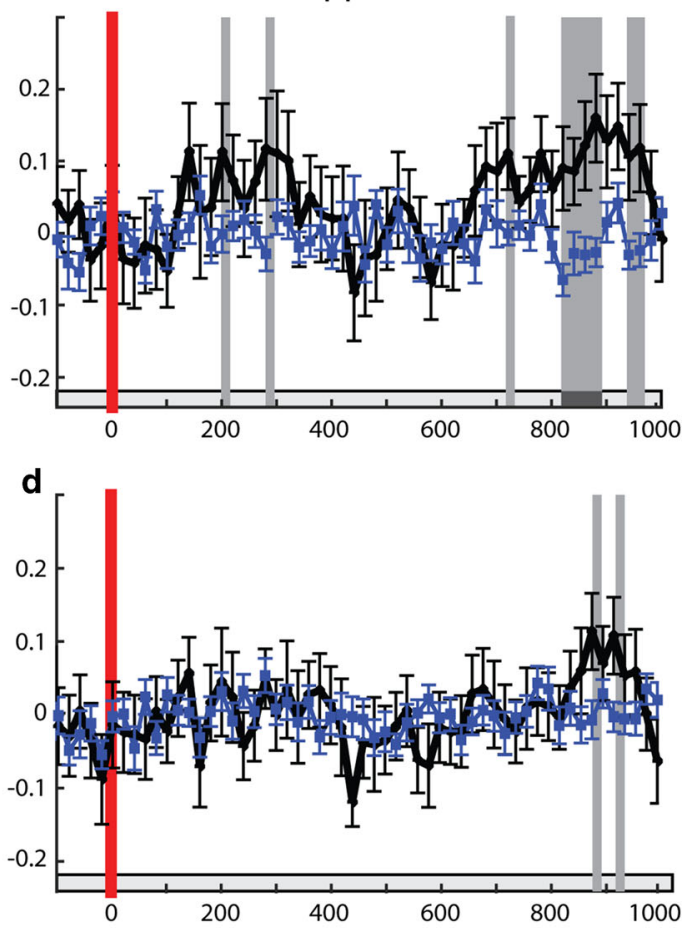

Fig. 2 Multivariate support vector regression results for anticipation phase. a Predicting success scores for sadness reduction via distraction. b Predicting success scores for sadness reduction via reappraisal. c Predicting success scores for disgust reduction via distraction. d Predicting success scores for disgust reduction via reappraisal. The black lines represent Fisher-transformed correlation coefficients between

additional analyses showing that only the strategy for which ER success was predictable during each phase was also decodable from the same neural patterns, suggesting that reappraisal involved stronger cognitive engagement in the anticipation phase, whereas for distraction, cognitive engagement was greater during implementation. Taken together, these results suggest that reappraisal benefits from fast anticipatory cognitive processes, while distraction does not. Distraction, on the other hand, appears to benefit more from a fast engagement of cognitive resources after an emotional stimulus has been presented, while the same is not true for reappraisal.

\section{Anticipation of emotion regulation}

Others have demonstrated the importance of anticipatory cues for ER, in particular when related to the intensity or the content of an upcoming stimulus (Shafir et al., 2015; Shafir \& Sheppes, 2018), or preparing for regulating versus not regulating one's emotions (Denny et al., 2014; Moser et al., 2009). The latter studies have revealed that neural responses can be systematically enhanced when preparing to use an ER strategy, particularly reappraisal. Our findings are in line with these reports, predicted and true labels (success scores). The blue line represents decoding results based on shuffled labels (empirical chance distribution for statistical testing). Grey vertical bars highlight significant time points $(p<.05)$; dark-grey sections of the horizontal bar at the bottom indicate time windows that additionally survived cluster correction. (Colour figure online)

suggesting that reappraisal success is to some degree related to the first second of cognitive processes recruited prior to its actual deployment. Given the very short time frame of these signals, this most likely does not reflect deliberate activation of resources or rehearsing of verbal strategies, but might indicate fast activation of a specific strategy schema that helps the regulator to implement the reappraisal strategy faster during implementation. This idea, however, is speculative at this stage, and one might have also expected a fast encoding of reappraisal success during the implementation phase as a consequence, which was not observed. Another option is that the results reflect the fast gathering of cognitive resources (see Di Russo et al., 2016; Van Boxtel \& Böcker, 2004) that help entering or "activating" a state that will set them up for overall success or failure in the upcoming implementation of reappraisal. Differences in neural patterns during this period might therefore reflect fluctuations in participants' reappraisal pre-activations. Alternatively, this result could also reflect fluctuations in prestimulus attention or general alertness, and it is possible that a state of hypervigilance influenced reappraisal success.

In contrast, distraction success was not related to early anticipatory activity cuing in the same way as reappraisal. At 
Implementation

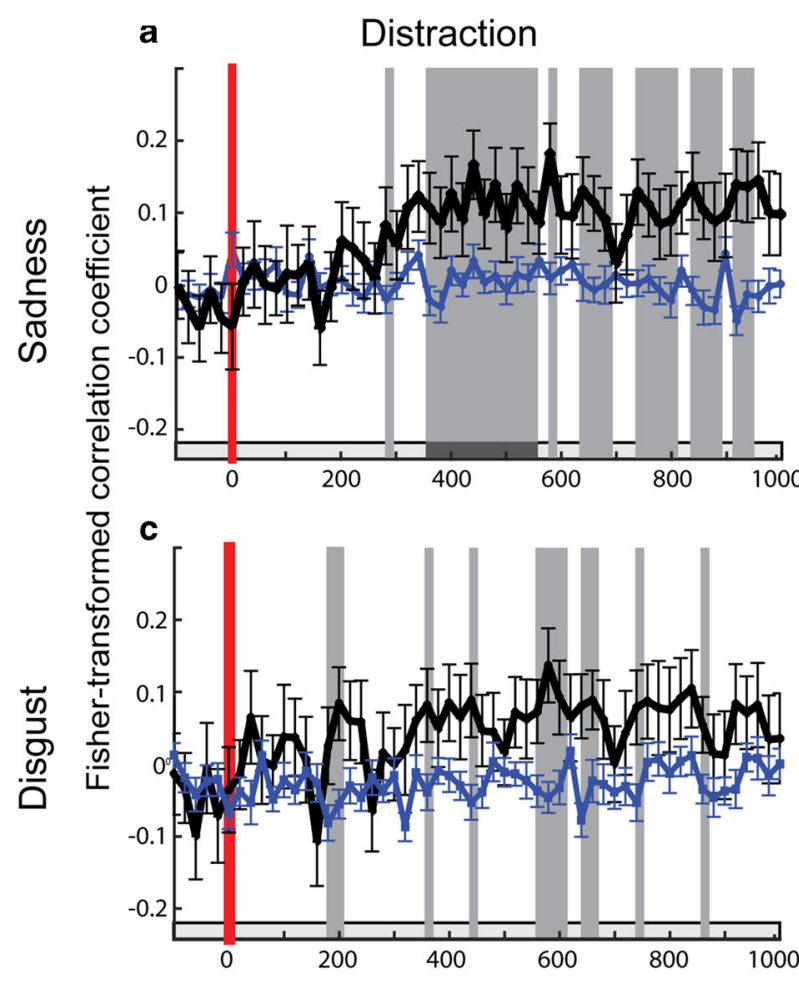

b Reappraisal

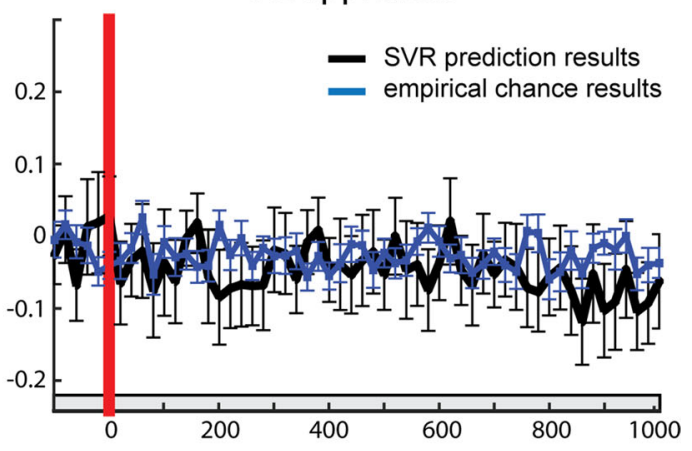

d

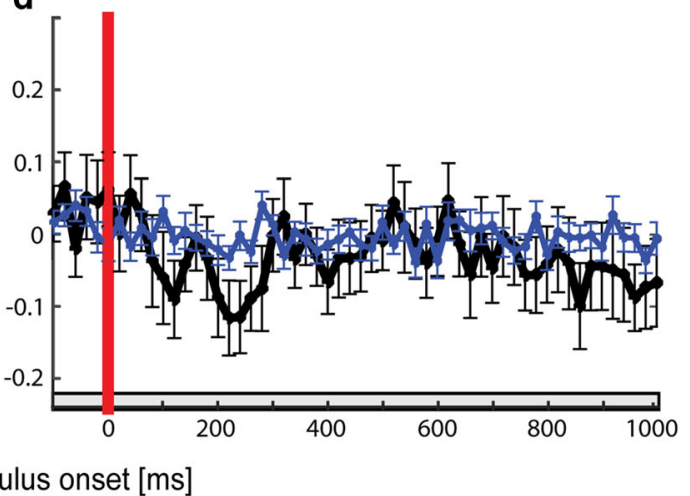

Fig. 3 Multivariate support vector regression (SVR) results for implementation phase. a Predicting success scores for sadness reduction via distraction. b Predicting success scores for sadness reduction via reappraisal. c Predicting success scores for disgust reduction via distraction. d Predicting success scores for disgust reduction via reappraisal. The black lines represent Fisher-transformed correlation coefficients between

first glance, this might appear counterintuitive because distraction could theoretically be prepared beforehand, given that the strategy could be applied in the same way for each stimulus (i.e., thinking about something unrelated to the emotional stimulus), while reappraisal requires unique, stimulus-specific implementations of the strategy (i.e., reinterpreting each specific emotional situation), which can only be fully developed once the stimulus is known. One possible explanation is that distraction is less cognitively demanding than reappraisal (Richards \& Gross, 2006; Shafir, Thiruchselvam, Suri, Gross, \& Sheppes, 2016; Strauss et al., 2016), meaning that regulators might not rely on engaging in mental preparation in the first-second postcue. Put otherwise, successful distraction may be less dependent on automatic preactivation of cognitive resources. If the results for reappraisal reflect fluctuations in attention or alertness, this difference in cognitive demand between the strategies may also explain why the reappraisal results did not generalize to distraction. As distraction is easier to implement, it may be that participants' levels of vigilance prior to regulating did not influence subsequent success to the same degree, and that processes occurring during distraction implementation were more important. predicted and true labels (success scores). The blue line represents decoding results based on shuffled labels (empirical chance distribution for statistical testing). Grey vertical bars highlight significant time points $(p<.05)$; dark-grey sections of the horizontal bar at the bottom indicate time windows that additionally survived cluster correction. (Colour figure online)

Our study is the first to focus on the first second of anticipatory brain activity to capture fast processes that did not require deliberate thinking. By doing so, we could investigate neural processes that were not susceptible to premature usage of strategies (e.g., starting to distract oneself before stimulus presentation) and clearly reflect the contribution of preparatory signals. MVPA, as implemented here, is superior when analyzing such fast processes, but not for sustained activation patterns. The reason is that later, deliberate preparation processes during anticipation would have strongly differed in their timing across trials and participants, meaning that with our small analysis time windows predictive signals would have heavily spread over time at the group level. Note, however, that our results do not preclude that distraction also benefitted from cognitive processes during the anticipation phase, but if so, these are likely to have occurred later.

\section{Implementation of emotion regulation}

During the first second of the implementation phase, it was possible to predict success for distraction in several time windows. ER success has been linked to ERP components 
(Hajcak \& Nieuwenhuis, 2006; Moser et al., 2009; Thiruchselvam et al., 2011) and shown to be related to neural patterns that also predicted reappraisal goals (Morawetz et al., 2016). The time points at which we could predict success for distraction in the current study were similar to distractionrelated late positive potential (LPP) component modulations in previous ERP studies (Schönfelder, Kanske, Heissler, \& Wessa, 2014; Shafir et al., 2015; Shafir \& Sheppes, 2018; Thiruchselvam et al., 2011). The relationship between neural activity and distraction success may reflect fluctuations in how well the strategy was executed in a given trial. Alternatively, it could also be due to trial-by-trial variations in effort, with less successful trials being those in which participants engaged less, or it might reflect fluctuations in attention, stimulus intensity, or other situational factors that we did not assess here.

Although reappraisal, like distraction, has been shown to modulate the LPP (Hajcak \& Nieuwenhuis, 2006; Moser et al., 2009), we could not predict reappraisal success when analyzing the first second after image presentation. However, both sadness and disgust could clearly be successfully regulated using both ER strategies, meaning that the implementation phase was of crucial importance for both. This means that reappraisal success in our study might have been more strongly linked to later cognitive processes rather than processes during the first second. There are at least two reasons to suggest this possibility. First, the two strategies have different underlying mechanisms with different temporal dynamics. Reappraisal should take more time to unfold because it requires the stimulus to be semantically processed first. In support, Thiruchselvam et al. (2011) found effects of reappraisal on the LPP only from 1,100 to $1,700 \mathrm{~ms}$ after stimulus onset. Distraction, however, can be applied immediately after the onset of the stimulus. Second, the strategies differ in how cognitively demanding they are (Richards \& Gross, 2006). Unlike distraction, implementation of reappraisal involves intricate processes (e.g., verbal labelling; Ochsner and Gross, 2008) and creativity (Wu, Guo, Tang, Shi, \& Luo, 2017). Reappraisal requires a unique reinterpretation of each emotional stimulus, making neural patterns during active implementation necessarily more varied, possibly precluding that the same clear, unified neural pattern emerged in each trial. Distraction, on the other hand, is simpler, requires similar cognitive processes each time it is implemented, and occurs earlier in the emotion generative trajectory (Richards \& Gross, 2006). This makes it more likely to find similar neural patterns during its implementation in each trial. In line with this, the general results for distraction were the same for both emotions. This supports that distraction could be implemented fast and independent of the specific stimulus, thereby reducing both negative emotions effectively.

\section{Limitations}

Although our results suggest that anticipation is important for reappraisal success, they do not provide conclusive evidence that it is not at all important for distraction, as there are several other factors that may have led to the null result-for example, there may be a relationship between distraction success and these early neural patterns that our method was not sensitive enough to detect. Our study may have lacked statistical power for the detection of very small effects, and there is currently need for methods of performing a power analysis for multivariate EEG studies. Alternatively, the time window analyzed may have been too early to detect effects, and it may be that cognitive processes occurring later in the anticipation stage are important for distraction, perhaps because distraction is less cognitively demanding than reappraisal is (Richards \& Gross, 2006). Future studies may examine the anticipation phase for distraction in more depth.

The present study examined only two discrete emotions. The regulation of other negative emotions, such as anger and fear, is also important for maintaining functioning (Cowen \& Keltner, 2017); therefore, future studies may examine a broader range of emotional experiences. Furthermore, in the present study, emotions were conceptualized as discrete entities, in order to minimize noise caused by interemotion differences in brain activity that may have occurred if general negative emotion had been used (Aftanas, Reva, Savotina, \& Makhnev, 2006). However, emotions are never entirely discrete (Cowen \& Keltner, 2017; Riegel et al., 2016). Hence, although the images selected for this task were high on either sadness or disgust, they naturally would have elicited some other emotions. Therefore, using ratings of general negative emotion may have produced stronger results (i.e., more variability in ratings, meaning that decoding may have been more effective).

\section{Conclusions}

Our results demonstrate the importance of cognitive preparation for the effective use of reappraisal. Reappraisal success could, to some extent, be predicted from neural patterns emerging in the first second after cuing for reappraisal, potentially related to activating cognitive resources or regulation tendencies and adapting an optimal mindset. Anticipatory processes therefore appear to play a crucial role in moderating processes of cognitive change when reinterpretation of an emotional situation is required. Our findings also open avenues for future work to explore the dynamic interplay between automatic and deliberate anticipatory processes, and how each of these contribute to the successful regulation of our emotions. 
Acknowledgements The authors thank Roni Shafir for valuable comments on the manuscript. This research was supported by a Melbourne School of Psychological Sciences Research Incentive Scheme Grant to S.B.; P.K. was supported by an Australian Research Council (ARC) Discovery Early Career Researcher Award (DECRA; DE190100203); C.M. was supported by a European Commission Marie SkłodowskaCurie Action Fellowship (795994).

Open practices statement Data and materials for the experiments reported here are available on request. The experiment was not preregistered. The analysis toolbox DDTBOX is freely available online.

Author contributions S.B., C.M., and P.K. designed the research. M.B. programmed the task. E.S. and J.A. conducted the research. E.S., J.A., M.B., and D.F. analyzed the data. S.B. and E.S. wrote the paper. All authors contributed to revisions of the manuscript and discussed the results.

\section{References}

Aftanas, L. I., Reva, N. V., Savotina, L. N., \& Makhnev, V. P. (2006). Neurophysiological correlates of induced discrete emotions in humans: An individually oriented analysis. Neuroscience and Behavioral Physiology, 36(2), 119-130.

Bagby, R. M., Parker, J. D. A., \& Taylor, G. J. (1994). The twenty-item Toronto Alexithymia Scale-1. Item selection and cross-validation of the factor structure. Journal of Psychosomatic Research, 38(1), 23 32.

Beauregard, M., Paquette, V., \& Levesque, J. (2006). Dysfunction in the neural circuitry of emotional self-regulation in major depressive disorder. NeuroReport, 17, 843-846.

Bode, S., \& Stahl, J. (2014). Predicting errors from patterns of eventrelated potentials preceding an overt response. Biological Psychology, 103, 357-369.

Bode, S., Bennett, D., Stahl, J., \& Murawski, C. (2014). Distributed patterns of event-related potentials predict subsequent ratings of abstract stimulus attributes. PLOS ONE, 9(10), e109070.

Bode, S., Feuerriegel, D., Bennett, D., \& Alday, P. M. (2019). The Decision Decoding ToolBOX (DDTBOX): A multivariate pattern analysis toolbox for event-related potentials. Neuroinformatics, 17(1), 27-41.

Chan, Y. M., Pianta, M. J., Bode, S., \& McKendrick, A. M. (2017). Spatio-temporal patterns of event-related potentials related to audiovisual synchrony jugdgments in older adults. Neurobiology of Aging, 55, 38-48.

Chang, C. C., \& Lin, C. J. (2011). LIBSVM: A library for support vector machines. ACM Transactions on Intelligent Systems and Technology, 2(3), 1-27.

Costa, T., Cauda, F., Crini, M., Tatu, M. K., Celeghin, A., de Gelder, B., $\&$ Tamietto, M. (2014). Temporal and spatial neural dynamics in the perception of basic emotions from complex scenes. Social, Cognitive and Affective Neuroscience, 9, 1690-1703.

Côté, S., Gyurak, A., \& Levenson, R. W. (2010). The ability to regulate emotion is associated with greater well-being, income, and socioeconomic status. Emotion, 10(6), 923-933.

Cowen, A. S., \& Keltner, D. (2017). Self-report captures 27 distinct categories of emotion bridged by continuous gradients. Proceedings of the National Academy of Sciences of the United States of America, 114(38), E7900-E7909.

Davis, E. L., Quiñones-Camacho, L. E., \& Buss, K. A. (2016). The effects of distraction and reappraisal on children's parasympathetic regulation of sadness and fear. Journal of Experimental Child Psychology, 142, 344-358.
De France, K., \& Hollenstein, T. (2017). Assessing emotion regulation repertoires: The Regulation of Emotion Systems Survey. Personality and Individual Differences, 119, 204-215.

Delorme, A., \& Makeig, S. (2004). EEGLAB: An open source toolbox for analysis of single- trial EEG dynamics including independent component analysis. Journal of Neuroscience Methods, 134(1), 921.

Denny, B. T., Ochsner, K. N., Weber, J., \& Wager, T. D. (2014). Anticipatory brain activity predicts the success or failure of subsequent emotion regulation. Social, Cognitive and Affective Neuroscience, 9(4), 403-411.

Di Russo, F., Lucci, G., Sulpizio, V., Berchicci, M., Spinelli, D., Pitzalis, S., \& Galati, G. (2016). Spatiotemporal brain mapping during preparation, perception, and action. NeuroImage 126, 1-14.

Dörfel, D., Lamke, J. P., Hummel, F., Wagner, U., Erk, S., \& Walter, H. (2014). Common and differential neural networks of emotion regulation by detachment, reinterpretation, distraction, and expressive suppression: A comparative fMRI investigation. NeuroImage, 101, 298-309.

Fink, J., Pflugradt, E., Stierle, C., \& Exner, C. (2018). Changing disgust through imagery rescripting and cognitive reappraisal in contamination-based obsessive-compulsive disorder. Journal of Anxiety Disorders, 54, 36-48.

Gross, J. J. (1998). The emerging field of emotion regulation: An integrative review. Review of General Psychology, 2(3), 271.

Gross, J. J. (2001). Emotion regulation in adulthood: Timing is everything. Directions in Psychological Science, 10(6), 214-219.

Gross, J. J. (2002). Emotion regulation: Affective, cognitive, and social consequences. Psychophysiology, 39, 281-91.

Gross, J. J. (2015). Emotion regulation: Current status and future prospects. Psychological Inquiry, 26, 1-26.

Gross, J. J., \& John, O. P. (2003). Individual differences in two emotion regulation processes: Implications for affect, relationships, and wellbeing. Journal of Personality and Social Psychology, 85(2), 348362.

Gross, J. J., \& Levenson, R. W. (1997). Hiding feelings: The acute effects of inhibiting negative and positive emotion. Abnormal Psychology, 106(1), 95-103.

Gyurak, A., Gross, J. J., \& Etkin, A. (2011). Explicit and implicit emotion regulation: A dual- process framework. Cognition \& Emotion, 25(3), 400-412.

Hajcak, G., \& Nieuwenhuis, S. (2006). Reappraisal modulates the electrocortical response to unpleasant pictures. Cognitive, Affective, \& Behavioral Neuroscience, 6(4), 291-297.

Holtzheimer, P. E., \& Mayberg, H. S. (2011). Stuck in a rut: Rethinking depression and its treatment. Trends in Neuroscience, 34(1), 1-9.

Houben, M., Van Den Noortgate, W., \& Kuppens, P. (2015). The relation between short-term emotion dynamics and psychological well-being: A meta-analysis. Psychological Bulletin, 141(1), 901-930.

Ivan, C. E. (2015). On disgust and moral judgments: A review. Journal of European Psychology Students, 6(1), 25-36.

Kanske, P., Heissler, J., Schönfelder, S., Bongers, A., \& Wessa, M. (2011). How to regulate emotion? Neural networks for reappraisal and distraction. Cerebral Cortex, 21, 1379-1388.

Kanske, P., Heissler, J., Schönfelder, S., \& Wessa, M. (2012). Neural correlates of emotion regulation deficits in remitted depression: The influence of regulation strategy, habitual regulation use, and emotional valence. NeuroImage, 61(3), 686-693.

Keltner, D., Sauter, D., Tracy, J., \& Cowen, A. (2019). Emotional expression: Advances in basic emotion theory. Journal of Nonverbal Behavior, 43, 133-160.

Kleiner, M., Brainard, D., Pelli, D., Ingling, A., Murray, R., \& Broussard, C. (2007). What's new in Psychtoolbox-3. Perception, 36(14), 1.

Koole, S. L. (2009). The psychology of emotion regulation: An integrative review. Cognition \& Emotion, 23(1), 4-41. 
Liu, Y., Lin, W., Xu, P., Zhang, D., \& Luo, Y. (2015). Neural basis of disgust perception in racial prejudice. Human Brain Mapping, $36(12), 5275-86$.

Marchewka, A., Żurawski, L., Jednoróg, K., \& Grabowska, A. (2014). The Nencki Affective Picture System (NAPS): Introduction to a novel, standardized, wide-range, high- quality, realistic picture database. Behavior Research Methods, 46(2), 596-610.

McRae, K., Hughes, B., Chopra, S., Gabrieli, J. D., Gross, J. J., \& Ochsner, K. N. (2010). The neural bases of distraction and reappraisal. Journal of Cognitive Neuroscience, 22(2), 248-262.

McRae, K., Ciesielski, B., \& Gross, J. J. (2012). Unpacking cognitive reappraisal: Goals, tactics, and outcomes. Emotion, 12, 250-5.

Morawetz, C., Bode, S., Baudewig, J., Jacobs, A. M., \& Heekeren, H. R. (2016). Neural representation of emotion regulation goals. Human Brain Mapping, 37(2), 600-620.

Morawetz, C., Alexandrowicz, R. W., \& Heekeren, H. R. (2017). Successful emotion regulation is predicted by amygdala activity and aspects of personality: A latent variable approach. Emotion, 17(3), 421-441.

Morawetz, C., Mohr, P. N. C., Heekeren, H. R., \& Bode, S. (2019). The effect of emotion regulation on risk-taking and decision-related activity in prefrontal cortex. Social Cognitive and Affective Neuroscience, 4(10), 1109-1118.

Moser, J. S., Hajcak, G., Bukay, E., \& Simons, R. F. (2006). Intentional modulation of emotional responding to unpleasant pictures: An ERP study. Psychophysiology, 43(3), 292-296.

Moser, J. S., Krompinger, J. W., Dietz, J., \& Simons, R. F. (2009). Electrophysiological correlates of decreasing and increasing emotional responses to unpleasant pictures. Psychophysiology, 46(1), $17-27$.

Oaten, M., Stevenson, R. J., \& Case, T. I. (2009). Disgust as a diseaseavoidance mechanism. Psychological Bulletin, 135(2), 303-321.

Ochsner, K. N., \& Gross, J. J. (2008). Cognitive emotion regulation: Insights from social cognitive and affective neuroscience. Current Directions in Psychological Science, 17(2), 153-158.

Ochsner, K. N., Ray, R. D., Cooper, J. C., Robertson, E. R., Chopra, S., Gabrieli, J. D., \& Gross, J. J. (2004). For better or for worse: Neural systems supporting the cognitive down- and up-regulation of negative emotion. Neuroimage, 23(2), 483-499.

Olatunji, B. O., Berg, H. E., \& Zhao, Z. (2017). Emotion regulation of fear and disgust: Differential effects of reappraisal and suppression. Cognition \& Emotion, 31(2), 403-410.

Posner, J., Russell, J. A., \& Peterson, B. S. (2005). The circumplex model of affect: An integrative approach to affective neuroscience, cognitive development, and psychopathology. Development and Psychopathology, 17(3), 715-734.

Richards, J. M., \& Gross, J. J. (2006). Personality and emotional memory: How regulating emotion impairs memory for emotional events. Journal of Research in Personality, 40(5), 631-651.

Riegel, M., Żurawski, Ł., Wierzba, M., Moslehi, A., Klocek, Ł., Horvat, M., ... Marchewka, A. (2016). Characterization of the Nencki Affective Picture System by discrete emotional categories (NAPS BE). Behavioral Research Methods, 48(2), 600-612.

Rivers, S. E., Brackett, M. A., Katulak, N. A., \& Salovey, P. (2007). Regulating anger and sadness: An exploration of discrete emotions in emotion regulation. Journal of Happiness Studies, 8(3), 393-427.

Russell, J. A. (1980). A circumplex model of affect. Journal of Personality and Social Psychology, 39, 1161-1178.

Schönfelder, S., Kanske, P., Heissler, J., \& Wessa, M. (2014). Time course of emotion-related responding during distraction and reappraisal. Social Cognitive and Affective Neuroscience, 9(9), 1310 1319.

Shafir, R., \& Sheppes, G. (2018). When knowledge is (not) power-The influence of anticipatory information on subsequent emotion regulation: Neural and behavioral evidence. Journal of Experimental Psychology: General, 147(8), 1225-1240.
Shafir, R., Schwartz, N., Blechert, J., \& Sheppes, G. (2015). Emotional intensity influences pre-implementation and implementation of distraction and reappraisal. Social Cognitive and Affective Neuroscience, 10(10), 1329-1337.

Shafir, R., Thiruchselvam, R., Suri, G., Gross, J. J., \& Sheppes, G. (2016). Neural processing of emotional-intensity predicts emotion regulation choice. Social Cognitive and Affective Neuroscience, 11(12), $1863-1871$.

Sheppes, G., \& Gross, J. J. (2011). Is timing everything? Temporal considerations in emotion regulation. Personality and Social Psychology Review, 15(4), 319-331.

Sheppes, G., \& Levin, Z. (2013). Emotion regulation choice: selecting between cognitive regulation strategies to control emotion. Frontiers in Human Neuroscience, 7, 179

Sheppes, G., \& Meiran, N. (2007). Better late than never? On the dynamics of online regulation of sadness using distraction and cognitive reappraisal. Personality and Social Psychology Bulletin, 33, 15181532

Sheppes, G., Catran, E., \& Meiran, N. (2009). Reappraisal (but not distraction) is going to make you sweat: Physiological evidence for self-control effort. International Journal of Psychophysiology, 71, 91-96.

Sheppes, G., Scheibe, S., Suri, G., \& Gross, J. J. (2011). Emotionregulation choice. Psychological Science, 22(11), 1391-1396.

Sheppes, G., Brady, D. J., \& Samson, A. C. (2014). In (visual) search for a new distraction: The efficiency of a novel attentional deployment versus semantic meaning regulation strategies. Frontiers in Psychology, 5, 346.

Siswandari, Y., Bode, S., \& Stahl, J. (2019). Performance monitoring beyond choice tasks: The time course of force execution monitoring investigated by event-related potentials and multivariate pattern analysis. NeuroImage, 197, 544-556.

Strauss, G. P., Ossenfort, K. L., \& Whearty, K. M. (2016). Reappraisal and distraction emotion regulation strategies are associated with distinct patterns of visual attention and differing levels of cognitive demand. PLOS ONE, 11, e0162290.

Szasz, P. L., Hofmann, S. G., Heilman, R. M., \& Curtiss, J. (2016). Effect of regulating anger and sadness on decision-making. Cognitive Behaviour Therapy, 45(6), 479-495.

Thiruchselvam, R., Blechert, J., Sheppes, G., Rydstrom, A., \& Gross, J. J. (2011). The temporal dynamics of emotion regulation: An EEG study of distraction and reappraisal. Biological Psychology, 87(1), 84-92.

Thompson, R. A. (1994). Emotion regulation: A theme in search of definition. Monographs of the Society for Research in Child Development, 59(2), 25-52.

Troy, A. S., Wilhelm, F. H., Shallcross, A. J., \& Mauss, I. B. (2010). Seeing the silver lining: Cognitive reappraisal ability moderates the relationship between stress and depressive symptoms. Emotion, 10(6), 783-795.

Turner, W. F., Johnston, P., de Boer, K., Morawetz, C., \& Bode, S. (2017). Multivariate pattern analysis of event-related potentials predicts the subjective relevance of everyday objects. Consciousness and Cognition, 55, 46-58.

Uusberg, A., Thiruchselvam, R., \& Gross, J. J. (2014). Using distraction to regulate emotion: Insights from EEG theta dynamics. International Journal of Psychophysiology, 91(3), 254-260.

Van Boxtel, G. J., \& Böcker, K. B. (2004). Cortical measures of anticipation. Journal of Psychophysiology, 18(2/3), 61-76.

Verduyn, P., \& Lavrijsen, S. (2015). Which emotions last longest and why: The role of event importance and rumination. Motivation \& Emotion, 39, 119-127.

Wager, T. D., Davidson, M. L., Hughes, B. L., Lindquist, M. A., \& Ochsner, K. N. (2008). Prefrontal-subcortical pathways mediating successful emotion regulation. Neuron, 59(6), 1037-1050. 
Webb, T. L., Miles, E., \& Sheeran, P. (2012). Dealing with feeling: A meta-analysis of the effectiveness of strategies derived from the process model of emotion regulation. Psychological Bulletin, 138(4), 775-808.

Wu, X., Guo, T., Tang, T., Shi, B., \& Luo, J. (2017). Role of creativity in the effectiveness of cognitive reappraisal. Frontiers in Psychology, 8,1598 .
Publisher's note Springer Nature remains neutral with regard to jurisdictional claims in published maps and institutional affiliations. 